A N N A L E S Annales de Bretagne et des Pays de l'Ouest

Anjou. Maine. Poitou-Charente. Touraine

108-4 | 2001

La Chambre des comptes de Bretagne

\title{
De la difficulté de réformer au XVIe siècle
}

l'échec du projet de suppression de la Chambre des comptes de Bretagne sous Charles IX

\section{Dominique Le Page}

\section{(2) OpenEdition \\ Journals}

\section{Édition électronique}

URL : http://journals.openedition.org/abpo/1681

DOI : $10.4000 / a b p o .1681$

ISBN : 978-2-7535-1484-3

ISSN : 2108-6443

Éditeur

Presses universitaires de Rennes

\section{Édition imprimée}

Date de publication : 20 décembre 2001

Pagination : 111-136

ISBN : 978-2-86847-674-6

ISSN : 0399-0826

Référence électronique

Dominique Le Page, "De la difficulté de réformer au XVIe siècle ", Annales de Bretagne et des Pays de l'Ouest [En ligne], 108-4 | 2001, mis en ligne le 20 décembre 2002, consulté le 02 mai 2019. URL :

http://journals.openedition.org/abpo/1681; DOI : 10.4000/abpo.1681 


\title{
De la difficulté de réformer $\mathrm{au} \mathrm{XVI}^{\mathrm{e}}$ siècle

\author{
l'échec du projet de suppression \\ de la Chambre des comptes de Bretagne \\ sous Charles IX
}

\author{
Dominique LE PAGE \\ Maître de conférences en histoire moderne \\ CRHMA-Université de Nantes
}

Les périodes d'affaiblissement du pouvoir royal sont en même temps des périodes de réformes où, dans un effort désespéré, les gouvernants tentent de mettre fin à ce qui a pu amener la crise. Ainsi les médiévistes ont-ils montré depuis longtemps l'importance de la première moitié du $\mathrm{XV}^{\mathrm{e}}$ siècle, en pleine guerre de Cent ans, tant sur le plan institutionnel qu'idéologique ${ }^{1}$; de même, aujourd'hui, les historiens modernistes s'intéressent-ils de plus en plus à la période des guerres de Religion. Henri III fait ainsi l'objet d'une réhabilitation en raison des changements dont il a été l'initiateur ${ }^{2}$; il en va de même pour son prédécesseur Charles IX qui, confronté aux premières guerres civiles, tenta, à l'instigation de son chancelier Michel de L'Hospital, de conjurer les périls en engageant non seulement une politique de concorde sur le plan religieux mais aussi une politique de rénovation de l'administration royale dans le but de résoudre les problèmes financiers auxquels le pays était confronté et de moraliser le gouvernement afin de mettre un terme à la colère divine. Cette ambition fut symbolisée par le tour de France réalisé par le roi à partir de 1564, au cours duquel la découverte des réalités locales s'accompagna d'un programme de réformes dont le couronnement fut constitué par la promulgation de l'ordonnance de Moulins en 1566. Du fait de la reprise des tensions religieuses, cette dernière ne put être véritablement appliquée et elle apparaît comme le symbole de l'incapacité du pouvoir à traduire sa volonté en actes face à une réalité complexe. L'analyse de la série B des Archives

1. Jacques KRYNEn, Idéal du prince et pouvoir royal en France à la fin du Moyen Âge (1380-1440), Paris, 1981.

2. Joël CORNETTE (sous la direction de), La monarchie entre Renaissance et Révolution, 1515-1792, Paris, 2000 et Nicolas Le RouX, La faveur du roi, Mignons et courtisans au temps des derniers Valois (vers 1547-vers 1589), Paris, 2000. 
départementales de Loire-Atlantique pour ces années a permis pourtant de constater que le mouvement réformateur engagé par Charles IX ne fut pas totalement sans effet dans le cas de la Bretagne puisqu'il se traduisit par une tentative sans précédent, de suppression de la Chambre des comptes : certes le projet n'aboutit pas mais il précipita l'institution dans une crise qui dura plus de deux années, dont il nous a semblé intéressant de restituer les péripéties et de dégager la signification.

\section{La suppression de la Chambre}

\section{Les circonstances}

Quand il parvint à Nantes le 11 octobre $1565^{3}$, Charles IX avait déjà accompli l'essentiel de son tour du royaume de France ${ }^{4}$ et sa visite, après trois journées passées dans la cité ligérienne, se limita pour l'essentiel à un séjour d'un peu plus de deux semaines à Châteaubriant, propriété du connétable de Montmorency, dont il repartit le trois novembre ${ }^{5}$. De la Bretagne, le roi vit donc peu de choses mais son ambition n'était pas d'en découvrir les différents aspects. Comme dans les autres provinces, la priorité était à la réforme et, dans ce sens, sa présence en Bretagne, aussi brève fût-elle, revêtit plusieurs significations. Elle voulut tout d'abord permettre l'application de décisions qui avaient été adoptées dans des étapes antérieures du voyage : il en alla ainsi de la réforme des justices locales qui permit, en regroupant plusieurs sièges de faible importance, de supprimer une série d'offices devenus inutiles. Esquissé lors du passage du roi à Troyes en 1564, relancé dans le Roussillon, ce projet trouva son aboutissement à Châteaubriant ${ }^{6}$. Une même démarche s'observe dans le contrôle des finances : dès juillet 1565, de Saint-Jean-de-Luz, le roi avait ordonné aux gens des comptes de Nantes de faire parvenir à son conseil plusieurs extraits de comptes rendus par des officiers en exercice dans la province ${ }^{7}$. L'opération se poursuivit à Châteaubriant où le roi institua un commissaire - Michel

3. Il fit son entrée officielle dans la ville le 12 octobre par la porte Saint-Nicolas. Les membres de la Chambre faisaient partie du cortège chargé de l'accueillir en compagnie d'officiers de finances qu'elle avait réquisitionnés pour l'occasion, notamment Pierre Cornulier, Jean Jallier, Robert Poulain, Antoine Charton, Jean Avril .... Arch. dép. de LoireAtlantique, B $584 \mathrm{f}^{\circ} 135$.

4. Jean Boutier, Alain Dewerpe, Daniel Nordman, Un tour de France royal. Le voyage de Charles IX (1564-1566), Paris, Aubier.

5 . Signe concret du passage du roi, en novembre 1565, les paroissiens de Saint-Jeande-Béré demandaient à être déchargés de deux feux et mettaient en avant, entre autres arguments, " la grande pauvreté dans laquelle ils sont et ont esté constituez à cause de la suite de la court qui y a séjourné ", Arch. dép. de Loire-Atlantique, B $584 \mathrm{f}^{\circ}$ 182-183.

6. Cf. Dom Charles TAILlandier, Histoire ecclésiastique et civile de la Bretagne, 2 vol., Paris, 1742-1746. Les justices de Touffou, de Loyaux et du Gâvre furent réunies à la juridiction de Nantes tandis que celles du Croisic, de Saint-Nazaire... étaient jointes à celle de Guérande.

7. Notamment de ceux qui s'étaient occupés de l'aliénation du domaine congéable dans la presqu'île de Rhuys, Arch. dép. de Loire-Atlantique, B 564 f f 122-124. 
Dessefort, conseiller au parlement - pour aller à la Chambre procéder aux extraits de documents nécessaires à l'examen des comptabilités en souffrance ${ }^{8}$. Engagé dans un mouvement de remise à plat de la gestion de ses officiers de finances, le monarque profita donc de sa présence dans la province pour faire remettre à ses conseillers toutes les pièces dont ils pouvaient avoir besoin dans le cadre des instructions en cours ${ }^{9}$.

Charles IX chercha aussi à régler sur place les problèmes les plus urgents. S'il se déplaça peu lui-même, il semble en effet avoir beaucoup reçu à Châteaubriant. Dans le cas de la Chambre des comptes, trois questions principales lui furent soumises qui concernaient le mode de fonctionnement interne de l'institution, l'étendue de ses attributions en matière de contrôle financier et surtout les conflits de juridiction avec le parlement. Sur le premier point, le roi, afin qu'une justice équitable fût rendue dans le domaine des finances, formula un règlement selon lequel toutes les décisions du Bureau de la Chambre devaient être prises en présence d'au moins six officiers, ce qui imposait le recours, en cas d'absence de présidents ou de maîtres, à des auditeurs. Sur le second point et contrairement au vœu des États de Bretagne, Charles IX confirma le droit de la Chambre à procéder au jugement de tous les deniers levés en Bretagne et particulièrement de ceux qui revêtaient un caractère extraordinaire comme les décimes, la solde des 50000 hommes et des francs-archers, les deniers d'octroi prélevés par les villes.

Face au conflit entre les deux cours souveraines, la situation était en revanche plus complexe. Depuis la mise en place d'un parlement permanent en 1554, ses membres étaient entrés en conflit avec les gens des comptes et contestaient le droit de ces derniers à juger les commis désignés pour faire la gestion des bénéfices saisis en régale et à juger des questions domaniales en première instance. Les parlementaires revendiquaient par ailleurs un rôle prépondérant dans les commissions en révision de comptes qui se tenaient à intervalles réguliers pour trancher sur les appels lancés par les officiers de finances contre les jugements rendus par la Chambre ${ }^{10}$. Derrière ces rivalités se dissimulaient bien entendu des questions d'épices, l'importance de celles-ci étant directement liée à l'étendue des affaires traitées par

8. Le roi prit également des décisions en matière de finances lors de son séjour à Châteaubriant : ainsi devant l'incapacité des fermiers des impôts et billots (ferme d'une valeur estimée à 160000 l) à fournir une caution, il ordonna que la perception des impôts serait assurée par les receveurs du fouage et que celle des billots serait prise en charge par le trésorier général Thévin, Arch. dép. de Loire-Atlantique, B 586 f f $^{\circ} 62$.

9. Celles-ci se poursuivirent après le départ du roi comme le suggère l'ordre donné au personnel de la Chambre le 19 novembre 1565 de faire compter les gens qui avaient reçu des deniers employés aux affaires de la guerre en Bretagne, Arch. dép. de Loire-Atlantique, $\mathrm{B} 583 \mathrm{f}^{\circ} 180$. En décembre 1565, le souverain demandait à la Chambre d'envoyer au Conseil privé des extraits des comptes rendus par le receveur général pour la vente des biens immeubles du clergé et du domaine ecclésiastique ainsi que la copie de tous les comptes de la recette générale depuis 1557, Arch. dép. de Loire-Atlantique, B 584 f $^{\circ} 201$.

10. Ces commissions étaient déjà attestées en Bretagne au Xve siècle, Jean KERHERVE, L'État breton aux $14^{e}$ et $15^{e}$ siècles, les ducs, l'argent et les hommes, Paris, 1986. 
les deux cours souveraines, mais également des questions d'honneur et de dignité. En demandant à juger en première instance les questions domaniales, la Chambre faisait non seulement appel au bon sens qui voulait que l'organe chargé de surveiller les receveurs ordinaires fût en même temps celui qui devait être saisi des éventuels problèmes d'usurpation mais tenait aussi à préserver une mission qui avait été à la base de sa création et qui lui permettait de participer à la défense de la présence royale en Bretagne; de même en revendiquant la parité dans les commissions en révision de comptes, elle ambitionnait de traiter sur un pied d'égalité avec le parlement.

L'importance de ces questions explique le véritable siège que la Chambre avait entrepris auprès du pouvoir royal à partir du début des années 1560 : en juin 1563, le second président Claude Méaucé et le procureur général Guillaume de Francheville avaient été envoyés au conseil privé pour exposer leurs doléances et demander un règlement identique à celui dont venait d'être pourvue la Chambre des comptes de Provence ${ }^{11}$; en juin 1564, dans la perspective de la venue du roi dans la province, une liste de remontrances lui était à nouveau adressée ${ }^{12}$ et quand Charles IX arriva à Nantes en octobre 1565, il reçut une délégation de la Chambre composée des premier et second présidents, du maître Jean Sorée et du procureur général. Cette pression amena le roi à organiser une confrontation entre des représentants des deux cours souveraines à Châteaubriant mais sans que cela débouche sur une ordonnance définissant avec précision leurs pouvoirs respectifs. Ce refus de trancher révèle sans nul doute une hésitation du pouvoir à prendre parti entre ses officiers; il suggère aussi que la présence des gouvernants dans la province, si elle devait permettre le dialogue, s'inscrivait dans une logique qui dépassait les intérêts particuliers et contribuait à l'élaboration de mesures embrassant l'ensemble du royaume.

Il en fut ainsi pour la Chambre des comptes qui, sans obtenir totalement gain de cause, avait tiré tout de même quelques satisfactions du passage du roi ; elle apprit pourtant sa suppression à la fin du mois de février 1566, au même titre que les autres Chambres des comptes de province ${ }^{13}$. Ordre fut aussitôt donné de transmettre à Paris la copie des comptes des trésoriers généraux ainsi que «tous les papiers, tiltres et enseignements, regis-

11. Arch. dép. de Loire-Atlantique, B 583 f ${ }^{\circ}$ 103-105.

12. On y trouve aux côtés des habituels griefs contre le parlement, des doléances plus générales concernant la mise à ferme du domaine à laquelle la Chambre était opposée, la difficulté pour obtenir paiement les officiers du droit de confirmation de leur charge... Certaines demandes furent satisfaites dans les années suivantes, ainsi celle qui portait sur la création de greffier à la Chambre ou celle qui demandait d'obliger les receveurs qui avaient levé des deniers avec l'autorisation du duc d'Étampes à comparaître à Nantes.

13. On peut supposer que cette mesure faisait partie de l'ensemble des décisions prises à Moulins en février 1566. Le texte de l'édit, qui a été conservé, et qui " portait règlement sur l'ordre et distribution des finances, suppression d'aucuns trésoriers de France, officiers comptables, suppression des Chambres des comptes de Dijon, Dauphiné, Provence, Montpellier, Nantes et Blois, et réduction des officiers de la Chambre des comptes de Paris au nombre ancien ", ne contient aucune mention de lieu ni de date, AN, F 23740 (253). 
tres et comptes et d'advertir les comptables de se retirer par devers les gens desdits comptes à Paris ${ }^{14}$ ". Le bilan du tour de France royal en matière de contrôle de finances conduisait donc à la suppression des instances locales et à une tentative de centralisation des moyens à Paris.

\section{Les causes immédiates de la suppression}

Si l'on s'interroge sur les raisons qui amenèrent le monarque à prendre une telle décision, il faut évoquer en premier lieu les principes qui guidaient l'action du pouvoir depuis le début des années $1560^{15}$. Pour faire face à la crise financière, la tendance était aux économies, ce qui passait, entre autres moyens, par une réduction du nombre des officiers. François II, dès le mois de mai 1560, avait ordonné la suppression de tous les offices créés dans les cours souveraines ${ }^{16}$ depuis le règne de Louis XII. La mesure fut appliquée puisque dans le cas de la Chambre des comptes de Nantes, on constate que les officiers décédés au début des années 1560 ne furent pas remplacés : cela fut le cas notamment de l'auditeur Julien Coué ${ }^{17}$ et des maîtres Guillaume Morin et René Bonnier. Remise en perspective, la mesure de suppression de la Chambre poussait donc jusqu'à son terme un effort de l'État pour contrôler ses effectifs ${ }^{18}$. Contrairement à des idées trop fréquemment enseignées, on voit que l'ambition de la monarchie au Xvi ${ }^{\mathrm{e}}$ siècle n'était pas de multiplier le nombre de ses agents et qu'il n'y avait pas contradiction, comme le fait remarquer Denis Crouzet, entre le principe du pouvoir absolu et celui d'une technostructure réduite ${ }^{19}$ : les officiers étaient encore considérés comme des charges pesant sur le peuple et comme des obstacles à une communication entre le roi et ses sujets.

À partir de l'accession de Michel de L'Hospital à la chancellerie, ce désir d'économie se doubla d'une volonté de moraliser l'État afin de mettre fin au péril religieux grandissant. Le nouveau chancelier était attaché à une justice exemplaire capable de mettre fin aux querelles et de soulager le peuple. Pour lui, l'important n'était pas d'avoir un personnel nombreux mais de disposer d'agents efficaces et vertueux, ce qui supposait un effort de discipline des officiers en place. Afin d'atteindre cet objectif, le pouvoir, sous sa houlette, rappela les règles en matière de service et chercha à com-

14. Arch. dép. de Loire-Atlantique, B $584 \mathrm{f}^{\circ} 236$.

15. Denis Crouzet, La sagesse et le malheur, Michel de L'Hospital, chancelier de France, Paris, 1998, p. 364 et suivantes.

16. Arch. dép. de Loire-Atlantique, B $117 \mathrm{f}^{\circ}$ 26-27.

17. Dont la charge, à la différence des autres officiers décédés, semble avoir été supprimée.

18. Cet effort de réduction du nombre des officiers ne concerna pas seulement les cours souveraines : dans le cas de la Bretagne, on constate en juin 1563 la réunion de l'office de trésorier des réparations à celui de trésorier général (Arch. dép. de Loire-Atlantique, B $583 \mathrm{f}^{\circ} 36$ ), la remise en cause des charges à caractère alternatif et une tentative de suppression des postes de receveurs ordinaires devenus presque inutiles depuis la mise à ferme des recettes.

19. Denis Crouzet, op. cit., p. 435 et suivantes. 
battre l'absentéisme; il renouvela aussi l'interdiction pour les officiers de servir d'autres maîtres que le roi ; il tenta enfin de légiférer en matière d'épices afin que la justice ne fût pas réduite à un commerce ${ }^{20}$. Loin là encore de demeurer au stade des vœux pieux, ces ambitions connurent des applications concrètes sur le terrain. Si l'on reprend l'exemple de la Chambre des comptes de Nantes, on remarque qu'en novembre 1563, l'institution enregistra le tarif établi concernant les sommes à payer par les personnes dont les causes étaient jugées par elle; plus concrètement des sanctions furent prises contre les greffiers qui prenaient des taxes trop élevées lors de la délivrance d'actes qu'il s'agisse de lettres d'hommage, d'attestations de serments de fidélité ou d'exemptions de feux de fouage ${ }^{21} . .$. En matière d'absentéisme, les officiers défaillants furent envoyés chercher à leurs frais pour venir siéger à la Chambre ${ }^{22}$ et les dispenses de service furent accordées avec beaucoup de réticence : ainsi le duc d'Étampes, gouverneur de Bretagne, dut-il intervenir à deux reprises auprès du Bureau de la Chambre en 1564 pour que le maître Pierre Gautier fût autorisé à s'absenter afin d'aller mettre fin aux troubles qui secouaient Guérande, ville dont il était le lieutenant ${ }^{23}$. Certains officiers semblent avoir été particulièrement sensibles aux attentes du pouvoir : c'est le cas du maître François Motay qui s'insurgea à plusieurs reprises contre les délégations trop nombreuses que l'institution envoyait auprès du roi, ce qui était source selon lui de dépenses multiples et inutiles alors que les finances étaient en crise ${ }^{24}$.

Tout semble donc confirmer que la Chambre ne fut pas insensible à l'esprit de réforme qui marquait le royaume au début des années 1560 : elle se conformait en cela à la volonté de l'État mais les exemples précédents révèlent que les représentants du roi en province en partageaient l'esprit ${ }^{25}$

20. L'ambition, très moderne, de Michel de L'Hospital était de mettre fin aux épices et de faire reposer la rétribution des officiers sur des gages revalorisés considérés alors comme un véritable salaire.

21. Le greffier qui commettait le plus d'abus, Jean Guilloppe, fut fait prisonnier sur ordre de la Chambre en mars 1564, Arch. dép. de Loire-Atlantique, B 583 f $^{\circ} 97$.

22. Ce fut le cas notamment le 7 mai 1563, Arch. dép. de Loire-Atlantique, B $583 \mathrm{f}^{\circ} 10$.

23. Arch. dép. de Loire-Atlantique, B $583 \mathrm{f}^{\circ} 74$.

24. Les requêtes de Motay entraînèrent un conflit avec le premier et le second présidents qui n'appréciaient pas ses critiques. Motay leur répliqua qu' « il s'émerveillait qu'on lui demande réparation pour lesdites remontrances veu... qu'elles ne tendent que pour la conservation des finances du roy et que c'est son office encores qu'il ne soit procureur du roy quand il voict les ordonnances de sadite majesté n'estre gardées ni observées de y tenir la main... ", mention du $1^{\mathrm{er}}$ juillet 1564, Arch. dép. de Loire-Atlantique, B $583 \mathrm{f}^{\circ} 196$ 198. Un an plus tard, en décembre 1565, après le passage du roi, Motay renouvelait ses accusations et s'élevait contre les trop fortes indemnités accordées aux membres de la Chambre qui avait été parler au roi à Châteaubriant, demandant le respect d'une ordonnance de Charles IX de juillet 1564, Arch. dép. de Loire-Atlantique, B 584 f 223.

25. Cet esprit perdura au moins chez certains officiers pendant plusieurs années : le 26 avril 1568, Martin de Beaune demandait ainsi à ce qu'on lui donne acte qu'il était venu de la ville de Tours, lieu de sa résidence, à Nantes " pour le service du roy et exercice de son estat et sur espoir de n'en partir jusques à trois moys que la présente ouverture sera finie suivant l'ordonnance gardée en la Chambre ", Arch. dép. de Loire-Atlantique, B $585 \mathrm{f}^{\circ} 226$. 
et qu'ils étaient persuadés que l'état de justice, en se rendant exemplaire, pouvait contribuer à résoudre les maux qui sévissaient alors. La situation pourtant était loin d'être idéale. Si l'on revient sur les problèmes d'absentéisme, on doit reconnaître que, malgré les rappels à l'ordre, les officiers, surtout dans les catégories des maîtres et des présidents, étaient toujours aussi peu empressés à venir faire service. On note bien entendu des différences de comportement entre les uns et les autres mais il était quasiment de règle qu'à chaque début d'ouverture, les tâches du bureau fussent assumées par les auditeurs en violation des ordonnances royales et en attendant que des maîtres, en nombre suffisant, voulussent bien se présenter. L'institution demeurait par ailleurs le lieu de nombreux conflits : conflits avec le parlement mais aussi entre les gens des comptes eux-mêmes. Le 15 mars 1564, le second président Claude Méaucé demandait ainsi réparation pour les injures que l'auditeur Geoffroy de Callac avait proférées contre lui « en plein Bureau luy séant en sa chère de président en face de justice et en présence de tous messieurs des comptes ${ }^{26}$ ». De Callac reprochait en fait à son supérieur d'avoir profité de son voyage à Paris en tant que représentant de la Chambre pour obtenir le consentement du pouvoir à la création d'une charge de greffier lésant ainsi les deux auditeurs qui assuraient jusqu'alors cette tâche. À la même période, les auditeurs entraient en rébellion ouverte contre une ordonnance royale de 1563 qui exigeait qu'ils examinent les comptes des receveurs, non plus à deux comme le voulait la coutume, mais seuls ${ }^{27}$. Cette mesure, qui visait à accélérer le traitement des comptabilités en souffrance, eut pour effet, au contraire, de plonger la Chambre dans une crise qui dura près d'un mois.

À ces tensions s'ajoutaient les problèmes de récusation qui perturbaient elles aussi l'activité de l'institution. On constate ainsi très souvent que les comptables ayant des difficultés pour justifier l'emploi qu'ils avaient fait des deniers prélevés par leurs soins récusaient un ou plusieurs des officiers chargés de les juger. L'argument le plus souvent invoqué était que l'officier incriminé représentait " leur plus mortel ennemi ": l'expression, pour sévère qu'elle paraisse, recouvrait en fait des réalités plus prosaïques comme des querelles d'argent, des inimitiés familiales ${ }^{28}$, des différends anciens à propos d'offices. Ces manœuvres avaient pour conséquence de retarder la rentrée des deniers dans les caisses royales; elles remettaient aussi en cause la capacité de la Chambre à rendre une justice équitable en

26. Arch. dép. de Loire-Atlantique, B $583 \mathrm{f}^{\circ} 107$.

27. Les auditeurs s'en prirent à ceux qui avaient rapporté les ordonnances royales à Nantes, à savoir le second président Méaucé et le procureur général Guillaume de Francheville, mais non au souverain lui-même qui demeurait inattaquable.

28. En mai 1564, le trésorier général Jacques Thévyn récusait le maître Jean Sorée en reprochant à ce dernier " d'être parent, allié et du conseil de Pierre Loppin, qui était son ennemi capital et d'être grand amy dudit premier président - Marc Fortia -, qui était pareillement son ennemy et que à ce moyen et aultres et autres causes qu'il avait cy devant baillées, il était haigneux et malveillant dudit Thévyn et que ledit Sorée luy avait escript pour les affaires de Loppin comme pour son intime amy ", Arch. dép. de LoireAtlantique, B $583 \mathrm{f}^{\circ} 151$. 
matière financière. Cela est net particulièrement dans le cas du premier président Marc de Fortia qui fut, au cours des années 1550 et 1560, récusé de façon systématique par les grands officiers de la province qui lui reprochaient tantôt ses malversations, tantôt son incapacité à exercer sa charge $^{29}$, ce qui contraignit les membres du Bureau de la Chambre à intervenir à plusieurs reprises pour " qu'on luy porte l'honneur et la révérance qui luy était deu ${ }^{30}$ ". Avec un tel président, la Chambre était loin de correspondre à l'idéal de Michel L'Hôpital d'officiers intègres et compétents capables de représenter dignement le roi.

On peut penser que le pouvoir prit conscience de tous ces problèmes lors de son séjour dans la province ${ }^{31}$ et la mesure de suppression décidée en février 1566, peut ainsi apparaître comme une tentative de surmonter les obstacles en se séparant de tous ceux qui n'étaient pas dignes de la confiance royale. En attribuant à la Chambre des comptes de Paris les prérogatives détenues jusqu'alors par ses homologues provinciales, on provoquait un regroupement de tous les moyens du contrôle financier sous l'œil même du roi à Paris : on retrouvait ainsi, d'une certaine manière, l'ambition des réformes financières du règne de François $\mathrm{I}^{\mathrm{er}}$ qui, pour mettre fin aux abus des comptables, avaient cherché à faire tomber directement tout le produit des impôts dans les caisses du roi pour que celui-ci puisse en connaître l'état et en disposer selon sa volonté ${ }^{32}$. En poursuivant un raisonnement comparable, Charles IX en arrivait à la conclusion qu'il ne pouvait y avoir de contrôle efficace des finances que par le biais des organismes qui siégeaient à proximité de lui afin qu'il puisse à tout moment surveiller les faits et gestes de son personnel. Ce faisant, il laissait supposer que le travail accompli jusqu'alors par ces intermédiaires tant décriés était inutile et il faisait le pari que le pouvoir pouvait se passer d'eux.

\section{Une Chambre des comptes inutile?}

La décision de suppression des Chambres de comptes de province ne procédait en effet pas seulement d'une volonté de l'État de réduire le nombre de ses agents, elle suggérait aussi que le travail effectué par ces derniers était de peu d'importance et qu'il pouvait faire l'objet d'une redistribution entre d'autres instances. Le pouvoir ne procéda probablement pas à une étude précise de l'activité des Chambres des comptes dans chacune

29. C'est le reproche que lui faisait Pierre Symonnot, contrôleur général des finances en Bretagne en juin 1561, Arch. dép. de Loire-Atlantique, B 581 f $^{\circ} 117$.

30. Arch. dép. de Loire-Atlantique, B 583 f $^{\circ} 118$.

31. Et ce d'autant plus qu'il avait reçu des plaintes d'officiers locaux : en décembre 1565, Jacques Budes, procureur général du parlement, avait adressé au conseil privé des remontrances contre la méthode suivie par la Chambre pour faire les extraits entre les parties, méthode qui, selon lui, " estoit plus pour le profit particullier de la compaignye que pour autre raison et que cela estoit contre le service du roy ", Arch. dép. de Loire-Atlantique, B $584 f^{\circ} 207$.

32. Philippe Hamon, L'argent du roi, les finances sous François $I^{e r}$, Paris, CHEFf, 1994. 
des provinces traversées mais l'on peut supposer qu'il connaissait suffisamment leurs interventions pour en arriver à une telle conclusion. Pour retrouver le regard porté par les responsables du temps sur les gens des comptes et estimer le travail effectué par ceux-ci, on dispose, dans le cas nantais, de deux sources principales : les livres des mandements royaux où sont consignés les édits et les ordonnances enregistrés par la Chambre, les livres des audiences qui mentionnent au jour le jour les questions qui ont été traitées par le bureau où siégeaient prioritairement présidents et maîtres. L'examen de ces deux sources pour l'année 1564, soit un an avant la venue du roi en Bretagne, amène à tirer plusieurs conclusions.

Tableau $n^{\circ} 1$ - Les mandements royaux enregistrés par la Chambre en 1564 (Arch. dép. de Loire-Atlantique, B 55)

\begin{tabular}{|lll|}
\hline Type d'actes enregistrés & & \\
\hline Institutions-provisions : & 1 & \\
$\quad$ - gouverneur de Bretagne & 1 & \\
$\quad$ - second président à la Chambre des comptes & 1 & \\
$\quad$ - maître maçon des villes et places fortes de Bretagne & 1 & \\
$\quad$ - payeur des gages des juges présidiaux de Rennes & 1 & \\
$\quad$ - garde et concierge d'Indrette & & $\mathbf{1}$ \\
Lettres de naturalité : & & $\mathbf{5}$ \\
Dons et pensions : & $\mathbf{8}$ \\
Lettres d'exemption d'impôts : & $\mathbf{3}$ \\
Lettres de rabais pour fermiers : & \\
$\quad$ Lettres de commission : & & $\mathbf{2}$ \\
Assignations sur recette de Bretagne : & $\mathbf{7}$ \\
$\quad$ - en faveur de créanciers du roi (Gondy, Obreth...) & 6 & \\
$\quad$ - pour paiement de gages & 1 & \\
Autre : & & \\
$\quad$ Lettre de ratification des prélèvements opérés & & $\mathbf{1}$ \\
$\quad$ par le gouverneur de Bretagne au temps des troubles & & $\mathbf{3 2}$ \\
\hline Total & & \\
\hline
\end{tabular}

Si l'on s'intéresse d'abord aux mandements présentés à la Chambre au cours de l'année 1564, on constate que celle-ci était amenée à donner logiquement son avis sur tout ce qui concernait les finances en Bretagne et qui pouvait avoir des incidences sur le travail de contrôle dont elle était chargée : elle était appelée ainsi à recevoir les serments des officiers responsables des principales recettes, à vérifier les actes de don, les rabais accordés aux fermiers mais aussi les assignations faites sur les impôts prélevés en Bretagne. De proche en proche, on voit que peu de domaines lui échappaient, de l'institution du gouverneur dont elle devait superviser le pouvoir d'ordonnancement des dépenses militaires à l'installation des étrangers dans la province par l'enregistrement des lettres de naturalité. La conjoncture pouvait l'amener à jouer un rôle plus important : ainsi en cette année 1564, où l'on pansait les plaies de la première guerre de Religion, dutelle donner son avis sur les remboursements à effectuer en faveur des 
créanciers du roi et dut-elle entériner, à la demande du souverain, les prélèvements autoritaires effectués par le duc d'Étampes en 1562 pour faire face aux troubles dans l'Ouest du royaume. L'examen attentif des différents mandements révèle par ailleurs que la Chambre n'était pas une simple cour d'enregistrement : gardienne des ordonnances royales, elle s'opposa à plusieurs reprises à des dons trop généreux du roi. Ainsi refusa-t-elle un temps d'enregistrer l'octroi du revenu du greffe civil de Rennes en faveur du seigneur de Bonnegarde en prétextant que le montant du don n'était pas précisé et que les receveurs en charge de la recette n'avaient pas rendu compte. Si elle dut finalement s'incliner face à la volonté royale, ce n'est qu'après que le roi eut confirmé son intention par la délivrance d'une lettre de jussion, ce qui lui permit en quelque sorte de dégager sa propre responsabilité. En cette période d'affaiblissement de la monarchie, la Chambre continuait donc à assumer sa double mission de relais du pouvoir central et de filtre des décisions prises au sommet.

L'analyse du livre des audiences conservé pour la même année permet d'affiner l'analyse : grâce à ce document, on peut en effet connaître le rythme de travail de l'institution et avoir un meilleur aperçu du champ de ses compétences puisque l'on y trouve mention de toutes les affaires traitées par le Bureau qui en était l'organe le plus important.

\section{Tableau $n^{\circ} 2$ - Le travail mensuel de la Chambre en 1564 \\ (Nbre : nombre de jours de travail)}

\begin{tabular}{|l|cccccccccc|c|}
\hline Mois & janv. & fév. & mars & avril & mai & juin & juil. & oct. & nov. & déc. & Total \\
\hline Nbre & 18 & 14 & 19 & 15 & 17 & 22 & 1 & 17 & 17 & 14 & $\mathbf{1 7 1}$ \\
\hline
\end{tabular}

Le tableau 2 montre ainsi qu'en 1564 la Chambre siégea 171 jours en respectant une interruption traditionnelle des travaux de juillet à septembre $^{33}$. Ce chiffre est plus élevé que celui des années ordinaires car les gens des comptes commencèrent à siéger dès le mois de janvier - au lieu de se retrouver au mois d'avril comme c'était la coutume - pour rattraper les journées de travail perdues en 1563 en raison de la peste qui sévissait alors à Nantes. En temps normal, l'institution devait se réunir entre 130 et 140 jours par an : cela peut paraître modeste mais correspondait à la norme des institutions d'Ancien Régime ${ }^{34}$. Si l'on rapporte le nombre de journées de travail à celui des affaires évoquées, on constate que les gens des comptes examinèrent en moyenne 3 dossiers par jour. Là encore l'activité ne semble pas très intense mais en l'absence de données sur le temps qui pouvait être consacré à chacun d'entre eux, il est difficile de tirer de cette donnée des conclusions pertinentes.

33. La Chambre observa également une "pause " entre le 24 mars et le 10 avril.

34. Ainsi le bureau de finances de Lyon analysé par Françoise Bayard siégeait en moyenne 93 jours par an sous le règne de Louis XIII, Françoise BAYARD, " Les activités du bureau des finances de Lyon dans la première moitié du XVII ${ }^{\mathrm{e}}$ siècle, " Colloque tenu à Bercy les 22 et 23 février 1996, L'administration des finances sous l'Ancien Régime, Paris, CHEFF, 1996, p. 242. 
La typologie des affaires traitées est en revanche plus riche d'enseignements. Le tableau 3 montre que les gens des comptes se préoccupaient de cinq grandes questions qui concernaient le fonctionnement interne de la Chambre, les questions judiciaires, la gestion du domaine royal, le contrôle des officiers de finances, l'enregistrement des actes du roi. Si l'on met à part cette dernière activité qui a été déjà évoquée lors de l'étude des mandements royaux ${ }^{35}$, on voit que les trois premiers secteurs mentionnés représentent une part équivalente - autour de $20 \%$ - nettement en dessous de la tâche de surveillance des comptables. En matière domaniale, la Chambre avait affaire surtout avec les catégories privilégiées ${ }^{36}$ de Bretagne en étant chargée depuis le règne de François $\mathrm{I}^{\mathrm{er}}$ de recevoir les hommages de la noblesse et les serments de fidélité des membres du clergé. Considérant cette tâche comme l'une de leurs plus importantes prérogatives, les gens des comptes l'exerçaient avec rigueur, en convoquant les récalcitrants devant eux et en mettant la saisie sur les biens de ceux qui refusaient obstinément de leur obéir. Un même soin s'observe en ce qui concerne les réformations : il n'y eut pas en 1564 d'opération de grande envergure comme cela s'était produit dans les années 1540 suite à l'ordonnance de Moulins ${ }^{37}$ mais les contrôles ponctuels furent menés, conduisant parfois, comme dans le cas de Rennes ou de Nantes, à la reprise de réformations anciennes non achevées. La vigilance des gens des comptes traduisait ici une inquiétude conjoncturelle, celle de voir remettre en cause par le parlement leur rôle de juge en première instance des questions domaniales. Chaque intervention était l'occasion de réaffirmer les droits de l'institution et de prendre date pour l'avenir.

\section{Tableau n ${ }^{\circ} 3$ - Les activités du Bureau de la Chambre en 1564}

(Arch. dép. de Loire-Atlantique, B 55; Total des actes : 524)

\section{Fonctionnement de la Chambre}

A. ouvertures-fermetures-clés

B. règlement interne

C. Auditeurs

Distribution de comptes

Nbre
1

6

35. Le plus grand nombre d'actes mentionnés dans le livre des audiences peut s'expliquer en partie par la négligence des greffiers qui ne tenaient pas à jour le livre des mandements.

36. Nous avons intégré dans l'activité domaniale un pouvoir original de la Chambre qui consistait à redistribuer des feux entre les paroisses de la province. La procédure était la suivante : des paroissiens pour des raisons diverses - pauvreté, calamité naturelle, guerre - présentaient une requête pour être déchargés d'un certain nombre de feux. Les gens des comptes, après enquête de l'un d'entre eux ou après consultation des receveurs du fouage des recettes concernées, prenaient une décision, généralement favorable mais souvent inférieure à ce qui était demandé, et redistribuaient les feux déduits sur les paroisses voisines, moins accablées par le sort. C'est la seule fonction de la Chambre qui la rendait "visible " pour la majorité de la population.

37. Dominique LE PAGE, Finances et politique en Bretagne au début des Temps modernes, 1491-1547, Paris, CHEFF, 1997. 
Dominique Le PAGE

$\begin{array}{lr}\text { Extraits } & 1 \\ \quad \text { Remontrances } & 3 \\ \text { Poursuites judiciaires } & 4 \\ \text { D. Interventions royales } & 7 \\ \quad \text { Lettres de cachet } & 1 \\ \text { E. Députation-commission-remontrances } & 5 \\ \text { F. Epices } & 1 \\ \text { G. Pensions } & 17 \\ \text { H. Taxes, jetons } & 6 \\ \text { I. Réparations. Petites dépenses } & 11 \\ \text { J. Excuses-dispenses de service-convocation personnel } & 10 \\ \text { K. Procureurs } & 14 \\ \text { L. Huissiers, sergents } & 8 \\ \text { K. Greffier } & 4 \\ \text { L. Menues nécessités } & 1 \\ \text { M. Attributions de la Chambre } & 4 \\ \text { N. archers, geôliers } & 2 \\ \text { Autre } & 96 \\ \text { Total } & \end{array}$

1 5

A. problèmes entre administrations

B. problèmes entre administration et particulier

C. problèmes entre particuliers

D. problèmes entre officiers

E. problèmes entre officiers et particuliers 22

E. demande et délivrance d'extraits, de copies 43

$\begin{array}{ll}\text { F. Autres } & 7\end{array}$

Total 106

\section{Domaine}

A. Aveux

Nbre \%

B. Serment de fidélité Hommage 13

C. Saisie 13

D. Main levée 4

E. Brefs 8

F. Réformation 14

G. Réparations, surveillance 4

H. Régale 3

I. Distribution de fouages $\quad 6$

Autre 12

$\begin{array}{ll}\text { Total } & 91\end{array}$

$\begin{array}{ll}\text { Contrôle du personnel des finances } & \text { Nbre }\end{array}$

A. Redditions. apurements $\quad 13$

B. Saisies-amendes $\quad 8$

C. Décharges, délais reddition 11

D. Prise de corps, emprisonnement, élargissement 11

$\begin{array}{ll}\text { E. Assignations, injonctions, ajournements } & 70\end{array}$

Déffailles 8 
F. Comparutions, interrogations 18

G. Exécutoires $\quad 3$

H. Récusations $\quad 11$

I. Commission révision comptes
Autres

Total $\quad 165$

Enregistrement

Nbre \%

A. Office

Enregistrement

Informations de vie et moeurs

Serments

Déboutements-délai

Autres

Lettres d'octroi

C. lettres d'anoblissement

D. Lettres de naturalité

E. Lettres de don-pension Enregistrement $\quad 26$

$\begin{array}{ll}\text { Renvoi } & 11\end{array}$

F. Lettres d'exemption

Enregistrement $\quad 6$

Renvoi 1

G. Commutation de deniers $\quad 1$

H. Paiement sur recette de Bretagne 5

I. Lettres de rabais 4

J. Contrats d'aliénation 2

Autre 3

Total $\quad 66$

Sur le plan judiciaire, la Chambre était occupée principalement par la délivrance d'extraits de documents - comptes, aveux et dénombrements, listes d'exempts à telle ou telle imposition, lettres d'institution à un office... - aux particuliers qui en avaient besoin pour la conduite de procès devant des instances de la province, parlement surtout mais aussi présidiaux. L'importance accordée par les gens des comptes à cette tâche se lit à travers le cérémonial qui entourait chaque délivrance d'extraits ${ }^{38}$; elle explique aussi les démarches réalisées pour réunir dans leurs bâtiments le plus grand nombre de documents - notamment les chartes des ducs alors conservées au château de Nantes - et les travaux engagés régulièrement pour en assurer une bonne conservation ${ }^{39}$. La Chambre, par les efforts déployés, par les sollicitations dont elle était l'objet, s'affirmait ainsi comme le centre d'archives principal de la province ${ }^{40}$. Faute d'avoir pu obtenir le

38. Elle se faisait en présence des parties concernées après que leur convocation solennelle à l'entrée de la Chambre eut été faite.

39. Là encore l'idéal poursuivi était loin d'être atteint : des feux se déclaraient dans les bâtiments, les clés des armoires se perdaient, les inventaires étaient rarement faits, des documents s'égaraient, une concurrence régnait entre les officiers pour savoir qui avait le pouvoir de procéder aux extraits.

40. La Chambre conservait ainsi précieusement les pancartes indiquant les prélèvements opérés sur les marchandises entrant ou sortant de Bretagne; elle possédait aussi 
droit de juger les différends entre les receveurs et leurs commis, faute aussi de pouvoir jouer un rôle la Chambre dans les litiges nés de la répartition de l'impôt, la Chambre voyait le reste de son rôle judiciaire, en-dehors de l'aspect purement comptable, cantonné à la résolution des problèmes entre les officiers de finances et des particuliers assignés sur eux ou entre les officiers eux-mêmes. La justice avançait à pas mesurés au rythme des ouvertures de l'institution, des absences ou des négligences du personnel mais aussi de celles des individus concernées par ces litiges. En ce domaine, la Chambre ne montrait ni plus de zèle ni moins de lenteur que les institutions comparables.

La troisième activité qui occupait les officiers du Bureau concernait le fonctionnement interne de la Chambre. On y a classé toutes les menues opérations qui permettaient la marche quotidienne de l'institution comme la gestion des épices, l'indemnisation des frais engagés par telle ou telle catégorie du personnel, les travaux à effectuer dans les bâtiments... On y a rangé aussi toutes les démarches découlant des relations entre la Chambre et le pouvoir central qu'il s'agisse des ordres donnés par le roi pour permettre l'application d'une décision ou, dans l'autre sens, des remontrances adressées par les gens des comptes au souverain. Tout cela faisait partie de l'ordinaire des jours et attestait que la Chambre, tant par les commandements qu'elle recevait que par les requêtes qu'elle présentait, demeurait un organisme vivant. Plus préoccupants étaient en revanche tous les débats suscités par les dysfonctionnements de l'institution : on y retrouve des problèmes déjà évoqués comme celui de l'absentéisme de certains officiers - ce qui nécessitait l'envoi de convocations, l'examen de lettres d'excuses -, les tensions au sein du personnel notamment entre les membres du Bureau et les auditeurs, ces derniers s'opposant à toute modification de leurs règles de travail, la difficulté enfin des gens des comptes à contrôler le personnel subalterne qui dépendait d'eux - procureurs des receveurs, sergents, archers, geôliers - et qui était indispensable à la bonne marche du travail de contrôle des receveurs, c'est à dire de ce qui constituait leur mission principale.

Avec plus de $30 \%$ des mentions, les opérations de surveillance des financiers constituaient la part la plus importante des affaires traitées. Ayant le pouvoir de contrôler la perception de tous les deniers royaux en Bretagne, les gens des comptes convoquaient devant eux tous les agents en activité, du trésorier général aux receveurs des décimes en passant par les officiers du fouage ou les commis à faire la gestion des biens saisis. En cette année 1564, signe de leur vigilance, ils assignèrent même à comparaître à Nantes les miseurs des cités qui avaient obtenu du roi le privilège de prélever des droits d'octroi, cherchant ainsi à soumettre à leur juridiction une catégorie qui y avait échappé jusqu'alors. Ces éléments positifs

un livre de réductions des mesures, ce qui permettait d'établir des équivalences entre les différentes unités utilisées dans la province, Arch. dép. de Loire-Atlantique, B 584 $\mathrm{f}^{\circ} 170$. 
doivent être cependant nuancés par deux constats. On a tout d'abord l'impression d'assister à une réduction du champ d'intervention de la Chambre : depuis la mise à ferme du domaine par Henri II, l'institution a ainsi perdu quasiment toute autorité sur les receveurs ordinaires et quand certains d'entre eux étaient convoqués pour rendre compte de ce qui demeurait de leur compétence, à savoir la perception des devoirs seigneuriaux et l'entretien des infrastructures, ils refusaient très souvent de comparaître. Le recours à l'affermage, la constitution de rentes sur les principaux revenus, l'assignation des créanciers du roi sur les différentes recettes tendaient ainsi à réduire le terrain d'intervention de la Chambre ${ }^{41}$. Celleci se montrait par ailleurs toujours aussi peu capable de faire payer les receveurs qui demeuraient redevables à l'égard du roi : de ce fait une grande part de son activité de contrôle consistait à lancer des assignations à comparaître. Parmi les dossiers instruits, peu étaient menés à terme : les héritiers du trésorier Florimont Le Charron, mort en 1561, se présentaient toujours régulièrement à la Chambre et le retard n'était-il pas dans ce cas encore trop catastrophique. Plus inquiétante est la mention de la convocation de descendants d'officiers ayant exercé au début du $\mathrm{XVI}^{\mathrm{e}}$ siècle comme Jean de Lespinay dont le passif de 80000 livres n'avait toujours pas été acquitté ou, mieux encore, à la période ducale comme Colinet de Marchy ou Jean Hagomar.

L'examen des livres des mandements et des audiences permet au final de faire un bilan mitigé de l'activité des gens des comptes de Nantes en cette année 1564 : du côté positif un rôle d'enregistrement des décisions royales assumé avec sérieux et une surveillance du domaine exercée avec un zèle d'autant plus actif que cette compétence était remise en cause par le parlement; du côté négatif, un fonctionnement interne laborieux marqué par les tensions et un contrôle somme toute peu efficace des finances. Ce bilan équilibré a toutefois quelque peu tendance à déformer la réalité telle qu'elle était perçue par le pouvoir royal : celui-ci devait être, en effet, davantage sensible à l'incapacité de la Chambre à faire payer les receveurs qu'aux autres aspects de son activité et c'est cette impuissance qui condamnait, à ses yeux, l'institution. Par ailleurs, aucune des fonctions assumées par la Chambre n'était incontestable, ce qui permettait d'envisager de les confier à d'autres instances. Ainsi l'enregistrement des actes pouvait être dévolu au parlement, la réception des hommages et des serments de fidélité aux présidiaux ${ }^{42}$, la surveillance du domaine aux cours inférieures et en appel au parlement, le contrôle des officiers à la Chambre des comptes de Paris.

41. Il est significatif à cet égard qu'une partie des remontrances adressées au roi par la Chambre en octobre 1565 critiquait la reconduction de la mise à ferme du domaine en 1561, les gens des comptes estimant les pertes subies depuis cette date à 100000 livres, Arch. dép. de Loire-Atlantique, B 584 f $^{\circ} 137$ et suivants.

42. Dès 1552, Henri II avait donné satisfaction à une remontrance des États de Bretagne qui voulaient que les hommages ainsi que les aveux et dénombrements pour les terres ayant un revenu inférieur à 100 l. de rente fussent rendus devant les présidiaux, Arch. dép. de Loire-Atlantique, B 584 f $^{\circ} 138$. 
Derrière la décision de supprimer la Chambre des comptes, on peut donc deviner l'esquisse d'un redécoupage cohérent de la carte administrative de la Bretagne - et du royaume - qui offrait tout à la fois l'avantage de mettre fin à une partie des tensions qui s'y développaient, de permettre une réduction du nombre des offices et donc de satisfaire les États provinciaux toujours soucieux d'économie, de répondre enfin à la préoccupation de l'ancien président des comptes qu'était Michel de L'Hospital de renforcer la Chambre de Paris face au parlement ${ }^{43}$. Avec le recul, la mesure de février 1566 pouvait paraître raisonnable et pourtant la suite des événements allait montrer que le projet, malgré un début d'application, ne pouvait être mené à son terme.

\section{L'échec de la réforme}

\section{Une tentative d'application}

Après l'annonce de sa suppression, la Chambre des comptes entra dans une période d'incertitudes et de flottement qui dura pendant plus de deux ans. Conscient de ne pas pouvoir mettre un terme brutal à l'institution, Charles IX précisa sa volonté par un édit donné à Saint-Maur-desFossés en juin 1566 par lequel il décida que les gens des comptes ne pouvaient désormais pas siéger à plus de quatre et qu'ils n'avaient plus le statut d'officiers mais de commis. En raison de cette concession, l'institution put poursuivre ses travaux. Elle continua à convoquer les comptables et à répondre aux sollicitations des administrés, notamment des paroissiens qui voulaient des rabais de fouage ${ }^{44}$. En mai 1567, quand les parlementaires rennais se présentèrent pour procéder à la révision des comptes, les formes habituelles furent respectées comme si de rien n'était ${ }^{45}$. La Chambre profita également de cette période d'incertitude administrative pour tenter de retrouver une certaine crédibilité : afin de montrer tout à la fois son utilité et son zèle à servir le roi, elle relança les opérations de réformation du domaine royal, prenant le soin dans le cas du domaine de Vannes de reproduire la totalité de l'acte qui l'amenait à intervenir afin de rappeler ses compétences en la matière; elle prolongea les ouvertures en juillet 1566 et en juillet 1567, la première fois en raison des " affaires qui s'y offraient ", la seconde par incertitude sur les intentions du roi et sur les contraintes que leur nouveau statut de commis leur imposait. Le monarque lui-même intervint pour orienter les travaux comme s'il voulait accorder une dernière chance à la Chambre : en novembre 1566, il leur ordonna ainsi d'obtenir paiement des sommes dues par

43. Robert Descimon, «Présentation », dans Michel de L'Hospital, Discours pour la majorité de Charles IX et trois autres discours, Paris, 1993, p. 17.

44. Les paroissiens de Saint-Jagu en l'évêché de Vannes bénéficièrent ainsi d'une remise de deux feux, Arch. dép. de Loire-Atlantique, B $585 \mathrm{f}^{\circ} 246$.

45. Parlementaires et gens des comptes recommencèrent à cette occasion leurs querelles pour savoir quelle institution devait fournir le plus de membres à cette commission, Arch. dép. de Loire-Atlantique, B 585 f 246. 
d'anciens receveurs comme Jean de Lespinay ou Laurent Pares qui n'avaient jamais soldé leurs comptes.

Ces initiatives ne parvinrent cependant pas à masquer la réalité d'une institution affaiblie dont les ordres n'étaient plus respectés. En mai 1566, le payeur Jean Avril refusa de remettre au second président François Le Bloy ce qui lui était dû, déclarant que son propre office avait été supprimé et qu'il n'était plus justiciable de la Chambre ${ }^{46}$. Un an plus tard, en juin 1567, le trésorier général Thévin, à qui était revenue la tâche de payer les gens des comptes, différa lui aussi le versement des gages de l'ensemble du personnel en arguant du fait que le roi avait ordonné de consacrer les fonds disponibles au paiement de la gendarmerie et au remboursement des plus gros créanciers de la monarchie. Il demanda par ailleurs à ce qu'on envoie son compte de la trésorerie à la Chambre de Paris comme ceux de ses prédécesseurs Florimont Le Charron et Aignan de Cailly et protestait de nullité contre ce qui serait fait contre lui à Nantes, affirmant " avoir occasion de récuser toute la compaignie pour ce qu'il n'y avoit aucun qui l'aimast, tesmoing la chanson " je le croy car tout le monde me le dict " et que ce qu'ils faisaient n'était que par animosité et pour desroger audit édict de suppression et maintenir leur juridiction contre icelluy édict et pour se venger des refus que fait ledit Thévin de leur bailler argent pour taxes qu'ilz se font les ungs aux autres contre les éditz et ordonnances du roy ${ }^{47}$ ".

Autre signe de défiance, mais cette fois de la part du roi, c'est l'envoi incessant de commissaires à Nantes. À la demande qui leur était faite en novembre 1566 de contraindre les anciens receveurs à s'acquitter de leurs dettes, les gens des comptes avaient en effet répondu qu'ils faisaient tout leur possible, que dès 1564 ils avaient mis la saisie sur les biens des héritiers de Lespinay mais que cette mesure avait été levée sur ordre du parlement ${ }^{48}$. Pour fondée qu'elle fût, cette argumentation n'avait pas de quoi satisfaire Charles IX ni de quoi le rassurer sur la capacité de la Chambre à examiner rapidement les comptabilités en instance : c'est pourquoi il fit saisir des archives de l'institution et ordonna leur transfert à Paris. Parmi les commissaires qu'il nomma à cette fin, on trouve des personnalités extérieures à la province comme le notaire et secrétaire royal Aymard Debabres ${ }^{49}$, les secrétaires du duc d'Anjou, Bernard de Girard, seigneur du Haillan qui était présent à Nantes aux mois d'avril et de mai 1566 et Renaud de Belvéder qui intervint en juillet et novembre 1567 mais aussi des officiers originaires de

46. Arch. dép. de Loire-Atlantique, B $585 \mathrm{f}^{\circ} 237$.

47. Idem, B 586 f $^{\circ} 91-92$. Malgré cette opposition, la Chambre décida de conclure son compte des réparations et de trancher les difficultés qu'il posait avant de l'envoyer à Paris. En conséquence de quoi, Thévin fit appel au conseil royal.

48. Le procureur fit valoir pour sa part qu'il avait fait rentrer dans les caisses royales plus de 40000 l. d'anciens deniers.

49. Celui-ci semble avoir été le premier commissaire royal envoyé à Nantes fin févrierdébut mars 1566 pour avoir communication des comptes de Jean Avril. Son échec aurait amené Charles IX à renouveler sa commission en faveur de Bernard de Girard qui arriva à Nantes dès le début de l'ouverture de la Chambre en avril 1566. 
Bretagne qu'il s'agisse du lieutenant civil de Nantes René Verge - il porta assistance au seigneur du Haillan en 1566 - de René de Bourgneuf, seigneur de Cucé, maître des requêtes de l'hôtel du roi, qui reçut pouvoir à la fin du mois de mai 1567 de saisir une nouvelle série de papiers ou de membres de la Chambre choisis en raison de la confiance que le roi leur accordait ${ }^{50}$.

D'après les indices fournis par les livres des audiences pour les années 1566 et 1567, on voit que l'attention du pouvoir se concentrait tout particulièrement sur des comptes " extraordinaires " qui, échappant aux mouvements de fonds traditionnels, étaient considérés comme porteurs de fraudes. Étaient ainsi concernés le compte de l'affranchissement du domaine congéable du domaine de Rhuys, celui des restes, celui des réparations des places fortes de Bretagne, celui des décimes ${ }^{51}$... Des receveurs figuraient également dans "l'œil du cyclone " comme le trésorier des États Jean Avril dont tous les comptes ${ }^{52}$ furent acheminés à Paris où l'on avait commencé à instruire son procès. En ligne de mire enfin les élites municipales nantaises qui durent justifier l'emploi des deniers d'octroi accordés par la monarchie depuis 1551 : des papiers furent saisis à cette fin à la Chambre des comptes mais aussi à la maison commune de la ville ${ }^{53}$.

Face aux commissaires, le personnel de la Chambre eut un comportement qui passa de la révolte à la résignation. Leur première réaction fut de tenter de résister aux demandes royales, c'est du moins ce qu'on peut déduire de la lettre de commission du seigneur de Haillan où le roi évoquait " les contumaces, reffus et remises d'aucuns officiers de notredite Chambre des comptes et dénonçait avec virulence une telle et si oultrecuydée désobéissance... qui démontrait ung monopolle faict par lesdits officiers pour par telz et sinistres moyens empescher que nous n'ayons claire et certaine cognoissance de la vérité qui nous faict présumer une intelligence que peuvent avoir lesdits officiers avec ledit Avril et sesdits complices, lesquelz officiers au lieu d'obéir à noz lettres se sont latitez et absentez, s'excusans

50. C'est le cas du second président François Le Bloy et du maître Jean Sorée qui furent chargés d'assister Renaud de Belvéder en novembre 1567, Arch. dép. de Loire-Atlantique, B $586 \mathrm{f}^{\circ} 160$.

51. Furent également transférés à Paris les comptes de l'abolition du convoi et de la suppression des juges présidiaux, celui de l'aliénation du temporel ecclésiastique, des impôts et billots, du retranchement des gages des officiers, des décimes, le premier compte de la recette des fouages de Cornouaille...

52. Le roi demanda de lui faire parvenir «toutes les charges et maniement qu'il avait euz en nostre dit pais et duché, soit des receptes particullières et fouages, paiement des officiers de notredite Chambre des comptes et générallement de toutes les charges et maniement qu'il a euz sans en excepter aucun ensemble les contrerolles de notre prévosté de Nantes et autres de Bretagne de 1552 jusqu'à 1556 ", Arch. dép. de LoireAtlantique, B $55 \mathrm{f}^{\circ} 275-278$. Suite à cette enquête, Avril fut incarcéré un temps à Paris, ce qui amena les États de Bretagne à se mobiliser en sa faveur.

53. C'est également Bernard de Girard qui fut chargé de saisir les papiers comptables de la municipalité nantaise dans la maison commune de la cité. Cette opération prenait place dans le mouvement de reprise en main des villes auquel le tour de France royal avait donné lieu et qui se traduisit notamment par un contrôle plus sévère des élections municipales, Arlette JouAnNA, La France du Xve siècle, 1483-1598, 1996, p. 422-423. 
les ungs sur les autres ${ }^{54}$ ». Les officiers incriminés étaient en fait une minorité où aux côtés d'un seul maître - Pierre Gautier, seigneur de Kerfur - figuraient surtout des auditeurs, à savoir Geoffroy de Callac, Pierre Riou, René Hachon, Pierre Ménardeau et Jean Guilloppe. Le roi leur interdit l'entrée de la Chambre pendant le temps de réalisation des extraits sous peine de privation de leur charge, révélant ainsi que la politique de rigueur dont était victime l'institution ne procédait pas seulement d'une accusation de lenteur dans le contrôle des finances mais aussi d'un soupçon de collusion avec les officiers qui avaient la responsabilité des principales recettes. Considérée sous cet angle, la mesure de suppression de la Chambre des comptes semble une conséquence directe de la mise en accusation des financiers, et de ce que l'examen de leurs comptes avait fait apparaître. On ne sait pourtant rien de la part de vérité qui se dissimulait derrière les accusations du roi et rien ne vient corroborer celles-ci par la suite ${ }^{55}$.

Pour leur part, les gens des comptes, passé ce temps de résistance, procédèrent aux extraits qui leur étaient demandés, tous rangs confondus $^{56}$, y consacrant notamment tout le mois d'avril 1566. Cette soumission à la volonté royale donne le ton de l'attitude qui fut la leur face aux exigences des autres commissaires : à peine intervinrent-ils pour que les formes fussent respectées, c'est à dire que les papiers soient délivrés en fonction de lettres patentes du roi et qu'un inventaire de tout ce qui était pris soit laissé à la Chambre. Placés en position d'accusés, ils durent assister sans mot dire à la remise en cause de leurs prérogatives : en octobre 1567, ils furent même contraints d'accepter que Renaud de Belvéder puisse assister à la reddition des comptes rendus à la Chambre et qu'il ait communication du rôle des restes dont il avait pouvoir d'assurer la perception. Leur position était d'autant plus fragile que leur nombre allait décroissant : aux offices restés vacants depuis le début des années 1560, vinrent se rajouter ceux des maîtres François Motay et Pierre de Chaurais et des auditeurs Pierre Riou, Geoffroy de Callac et Tristan de Saint-Martin qui ne furent pas remplacés après leur décès, ce qui réduisit les effectifs de la Chambre à deux présidents (Fortia et Le Bloy), à quatre maîtres (Pierre Gautier, Jean Sorée, Pierre de Francheville, Martin de Beaune), quatre auditeurs et un procureur général. Avec ces évolutions, on retombait donc net-

54. Arch. dép. de Loire-Atlantique, B 55 f $^{\circ} 275$.

55. Le seul officier pour lequel on réussisse, au stade actuel des recherches, à établir des liens avec Jean Avril est René Hachon qui reconnut quelques années plus tard avoir été son commis à Ancenis, lieu dont il était originaire, pour la ferme de la prévôté de Nantes. Sans qu'il y eût forcément complicité entre les gens des comptes et Jean Avril, il y avait à tout le moins une certaine solidarité puisque ce dernier était le payeur de leurs gages.

56. À l'exclusion toutefois des gens qui avaient été écartés par le roi. D'après une liste d'indemnisation établie le 21 juin 1567, on sait que les présidents Marc de Fortia, François Le Bloy, les maîtres Martin de Beaune, Jean Sorée, Pierre de Francheville, le procureur général Guillaume de Francheville, l'auditeur Alexandre de La Tullaie et le garde Guillaume Meneust travaillèrent pendant un mois pour effectuer les extraits demandés, Arch. dép. de Loire-Atlantique, B 585 f $^{\circ}$ 76-77. 
tement en-dessous des effectifs originels de la Chambre et l'on trouve une nouvelle confirmation de la volonté du pouvoir de parvenir à une extinction que l'on pourrait qualifier de naturelle de cette dernière.

L'obstination n'était pourtant pas la qualité maîtresse de la monarchie en ces années où le pouvoir vacillait et en août 1568, le général des finances Nicolas de Troyes présentait à la Chambre un édit royal révoquant les décisions prises en 1566. Peu de temps après, tous les officiers décédés furent remplacés (tableau $n^{\circ} 4$ ) et l'institution retrouva la totalité de ses pouvoirs.

Tableau $n^{\circ} 4$ - Nominations à la Chambre en 1568-1569 (Source : Arch. dép. de Loire-Atlantique, B 586)

\begin{tabular}{|c|c|c|c|c|}
\hline & Fonction & Date & Précédent titulaire & Références \\
\hline Pier & re & 25.10 .1568 & François Motay & B $586 f^{\circ} 61$ \\
\hline Jean Jallier & maître & 29.11 .1568 & René Bonnier & B $586 \mathrm{f}^{\circ} 120$ \\
\hline Marc de Barbère & maître & 30.09 .1568 & Pierre de Chaurais & B $586 \mathrm{f}^{\circ} 123$ \\
\hline Antoine Charreton & maître & 30.09 .1568 & Guillaume Morin & B $586 \mathrm{f}^{\circ} 123$ \\
\hline François Symon & auditeur & 01.10 .1568 & Julien Coué & B $586 \mathrm{f}^{\circ} 123$ \\
\hline Guillaume Gougeon & auditeur & 28.12 .1568 & Geoffroy de Callac & B $586 \mathrm{f}^{\circ} 269$ \\
\hline François de Bruc & auditeur & 07.05 .1569 & Pierre Riou & B $586 \mathrm{f}^{\circ} 193$ \\
\hline Jean Picault & auditeur & 23.05 .1569 & Jean Jallier & B $586 \mathrm{f}^{\circ} 193$ \\
\hline
\end{tabular}

\section{La réforme impossible}

Pour comprendre le revirement d'attitude du roi, différents éléments doivent être pris en compte. Le plus important d'entre eux est bien entendu la reprise des guerres de Religion après la surprise de Meaux en 1567 : confrontée de nouveau à la nécessité de trouver des quantités importantes d'argent pour financer les opérations militaires, la monarchie fut contrainte de recourir aux expédients habituels et notamment aux ventes d'offices. En Bretagne, Charles IX réintroduisit, en cette même année 1568, une deuxième charge de trésorier général, rétablit les postes de receveurs alternatifs du fouage et restaura à titre d'office des fonctions qui avaient été réduites au cours des années précédentes au rang de commission comme celle de contrôleur général des finances bretonnes. La nomination de nouveaux titulaires à la Chambre des comptes s'inscrivit donc dans un mouvement plus large de remise en cause des mesures d'économie qui avaient été adoptées jusqu'alors.

Ces éléments du contexte général ne doivent pas amener à négliger les autres facteurs qui contribuèrent à faire échouer le projet royal. Il faut d'abord mentionner que la Chambre refusa toujours, malgré les atteintes qui lui étaient portées, de reconnaître sa suppression : ainsi quand le sénéchal de Nantes tenta les 20 et 28 novembre 1566 de mettre fin à son activité, lui demanda-t-elle de surseoir à son action tant qu'elle n'avait pas présenté ses doléances à Charles $\mathrm{IX}^{57}$. Dans la pratique, elle délégua à deux reprises

57. Idem, B $584 \mathrm{f}^{\circ} 384$. 
plusieurs de ses membres auprès du roi : une première fois, en mai 1566, pour lui exposer les inconvénients qui résulteraient de la suppression de l'institution ${ }^{58}$, une seconde fois en décembre 1566 pour s'opposer à la réduction du personnel et à la transformation des offices en commissions ${ }^{59}$. Raisonnant au nom d'une idéologie traditionnelle qui voulait qu'aucune décision du monarque ne fût appliquée tant que les remontrances des cours souveraines n'avaient pas été entendues, la Chambre considéra la décision de Charles IX, du fait des démarches qu'elle avait entreprises, comme nulle et non avenue. Ce raisonnement lui permettait en août 1568, quand Charles IX la rétablit dans ses pouvoirs, de refuser de payer un quelconque droit de confirmation pour les offices existant encore à cette date en s'appuyant sur le fait que l'édit de suppression n'avait " esté receu ny publié audit pays selon les antiens privillèges et ordonnances dicelluy et confirmez par sadite majesté, aussi peu y a-t-il esté satisfait par eux qui, au contraire, ont toujours continué à faire juc à présent leur exercice au service de sadite majesté tout ainsi qu'ils l'ont ordinairement accoutumé ${ }^{60} \ldots$.."

Dans son opposition à la politique de Charles IX, la Chambre reçut par ailleurs plusieurs soutiens dont celui des autres cours des comptes également menacées par l'édit de suppression. Les gens des comptes envoyés à Paris en décembre 1566 eurent pour tâche de prendre contact avec les délégués des Chambres de Bourgogne et de Provence afin d'envisager une riposte commune ${ }^{61}$. L'offensive royale eut ainsi pour effet de développer la solidarité entre les membres des cours souveraines de différentes provin$\operatorname{ces}^{62}$ et de contribuer à renforcer l'identité du groupe des officiers face à la monarchie. En Bretagne, les gens des comptes bénéficièrent de l'appui de différentes instances parmi lesquelles on doit mentionner en premier lieu la municipalité nantaise qui écrivit au roi pour protester contre l'édit de suppression. Encore mal remis de la perte du parlement survenue en

58. Ce furent l'auditeur Golven de Kerchrist - il était licencié en lois - et le docteur et procureur général Guillaume de Francheville qui furent envoyés en délégation à Paris le 2 mai 1566. Ils y séjournèrent au cours des mois de juin, juillet et août. Francheville était chargé aussi de défendre les intérêts de la Chambre contre les accusations portées contre elle devant le conseil privé par le maître François Motay, Arch. dép. de Loire-Atlantique, B $584 \mathrm{f}^{\circ} 306-307$.

59. Le président Marc de Fortia et Pierre Gautier, «l'un des plus anciens maîtres " ainsi que l'auditeur René Hachon furent cette fois adjoints au procureur général Guillaume de Francheville pour empêcher l'application de l'édit adopté à Saint-Maur-des-Fossés en juin 1566, Arch. dép. de Loire-Atlantique, B 584 f $^{\circ} 351$.

60. Idem, B $586 \mathrm{f}^{\circ} 118-119$.

61. Id., B $584 \mathrm{f}^{\circ} 351$. On assisterait ainsi pour la première fois dans l'histoire de la monarchie à la mise en place d'une alliance entre les officiers du roi pour défendre leur statut, préfiguration de ce qui allait se produire au XVII ${ }^{\mathrm{e}}$ siècle et plus encore au XVIII ${ }^{\mathrm{e}}$ siècle. Cette remarque demandera à être vérifiée en analysant ce qui s'est produit à la même période dans les Chambres des comptes de Bourgogne et de Provence.

62. Plusieurs indices inclinent à penser qu'il existait dès le $\mathrm{XVI}^{\mathrm{e}}$ siècle une correspondance régulière entre les membres des différentes Chambres des comptes du royaume : cela contribuait à uniformiser les modes de fonctionnement et à développer les actions pour l'obtention des mêmes privilèges. 
1561, les notables nantais, malgré les différends qui les opposaient aux gens des comptes dans le processus de mise en place d'un corps de ville ${ }^{63}$, insistèrent principalement sur le fait que cette mesure reviendrait à priver leur cité de toute institution d'" ancienne authorité et décoracion ${ }^{64}$ " et demandèrent donc au roi de la reconsidérer. Leur protestation fut amplifiée par celle des États de Bretagne qui adressèrent une lettre solennelle de remontrances à Charles IX ${ }^{65}$. Leur argumentation se développait en plusieurs points. Ils insistèrent d'abord sur le fait que la suppression de la Chambre constituait une violation des privilèges de la province, privilèges qui avaient été confirmés par les prédécesseurs de Charles IX depuis 1491 et qui stipulaient que les Bretons ne pouvaient " pour aucune cause estre tirez hors icelluy et pour ceste raison y a eu de toute ancienneté ung parlement, court souveraine au faict de la justice, et ladite Chambre pour les finances, en laquelle tous ceux qui touchent deniers levez audit pays d'autorité du roy, sont tenus compter et non ailleurs".

Ils mirent ensuite en avant les attributions de la Chambre - conservation des archives de la province, réception des aveux, redistribution des fouages, contrôle du personnel des finances - en insistant à chaque fois sur les services irremplaçables que l'institution rendait aux différentes composantes de la population bretonne et sur les économies qu'elle leur permettait de réaliser en évitant de longs déplacements pour faire enregistrer des lettres de don, pour obtenir un extrait d'acte ou pour présenter un compte. Les gens des États rejetèrent ensuite l'idée que la suppression de la Chambre pouvait permettre à la monarchie de diminuer ses dépenses de façon substantielle en rappelant que la majeure partie des officiers de finances de Bretagne rendaient leurs comptes à leurs propres frais et que le travail de contrôle n'entraînait donc pas de surcoûts pour le roi. Ils soulignèrent aussi que les gages des gens des comptes " estoient fort petits eu esgard au service qu'ils font à sa majesté et audit pays, se montans en tout que huit à neuf mille livres par chacun an et se paient sur les deniers qui sont levez audit pays ${ }^{66}$ " et n'hésitèrent pas à affirmer que depuis la création de la Chambre et grâce à son action, les revenus de la Bretagne étaient passés " par chacun an de 150 ou au plus 300000 livres à 1 million de liv-

63. François II avait accordé la création d'un corps de ville à Nantes en 1559 mais du fait de l'opposition conjuguée des différentes cours de justice présentes dans la cité dont la Chambre des comptes -, des autorités religieuses, de l'Université et du gouverneur René de Sanzay, le projet ne put aboutir avant le mois d'octobre 1564, date à laquelle Charles IX confirma les intentions de son prédécesseur. Le premier maire de Nantes fut élu le 28 novembre 1564, Guy SAuPIN, La vie municipale à Nantes sous l'Ancien Régime, 1565-1789, thèse pour le doctorat de troisième cycle, dactyl., Nantes, 1981, p. 16.

64. Arch. municipales de Nantes, II 13, acte du 15 novembre 1566.

65. Arch. dép. d'Ille-et-Vilaine, C 3720.

66. Ils prenaient soin de préciser que cette somme était prélevée sur une taxe qui n'avait pas d'équivalent dans les autres provinces, en l'occurrence les brefs, sorte d'assurances qui étaient délivrées aux marins naviguant au large de la Bretagne et qui leur permettaient de récupérer leur cargaison en cas de naufrage. Les brefs étaient scellés à la Chambre des comptes. 
res, sans compraindre les deniers extraordinaires ". La solution intermédiaire qui consistait à réduire le nombre des gens des comptes et à les ravaler au statut de commis leur paraissait elle aussi inadmissible dans la mesure où elle ne permettait pas à la Chambre de faire face à ses différentes missions avec suffisamment de rapidité ${ }^{67}$ et donc de répondre aux besoins du pays " et des pauvres subiectz dicelluy, lesquelz s'ilz en estoient privez ou qu'ilz n'eussent moyen d'avoir prompte expédition en ladite Chambre comme ilz auroient au passé, seroient grandement endommaigez et intéressez ".

Défense de l'intérêt public, insistance sur les impacts financiers mais aussi pratiques de la suppression, attachement aux privilèges de la province étaient au final les maîtres mots de l'argumentation des gens des États. Sans que l'on sache la part que ces remontrances eurent dans le changement d'attitude de la monarchie, on peut souligner tout du moins qu'elles mirent en évidence les maladresses de cette dernière. Les gouvernants semblent ainsi avoir sous-estimé la force de l'attachement aux privilèges de la Bretagne : aussi bien la ville de Nantes que les États prirent la défense de la Chambre des comptes parce qu'elle faisait partie des institutions traditionnelles de Bretagne reconnues officiellement par la monarchie lors de l'intégration de la principauté au royaume et que son existence ne pouvait donc être remise en cause. Le recours à cet argument amena le roi à demander aux gens des comptes de lui adresser une copie des contrats de mariage conclus par ses prédécesseurs pour en vérifier le fondement ${ }^{68}$ et explique peut-être pourquoi il décida entre février et juin 1566, de passer de la décision de supprimer la Chambre à celle de réduire ses effectifs, sans parvenir toutefois à satisfaire les élites provinciales puisque celles-ci étaient non seulement attachées à l'institution mais aussi à une composition précise qu'elles considéraient comme une donnée intangible. La même " légèreté " du pouvoir se lit dans la conduite de l'opération de suppression de la Chambre : rien ne semble avoir été fait pour confier rapidement ses attributions à d'autres institutions, ce qui contraignit la monarchie à continuer à recourir à ses services - démontrant ainsi son utilité - et permit aux États de souligner les inconvénients que la réduction du personnel entraînait. La monarchie eut le tort enfin, en multipliant ses opérations de contrôle - à destination des villes, à destination du trésorier des États dont on a vu qu'il fut emprisonné - de s'aliéner le soutien de la majeure partie des élites provinciales et de créer contre elle un front du refus qui se cristallisa autour de la défense de la Chambre des comptes.

67. Ils mentionnaient précisément le cas des commissions en révision de compte qui ne pourraient plus se tenir si la Chambre n'était pas en mesure de fournir un nombre de membres équivalent à celui des gens du parlement.

68. Le 6 juin 1566, copie fut faite par les gens des comptes des lettres de mariage entre Anne de Bretagne et Louis XII, entre François ${ }^{\text {er }}$ et Claude; elle fut transmise au roi par René de Bourgneuf, seigneur de Cucé, qui avait été commis à cet effet, Arch. dép. de LoireAtlantique, B 584 f $^{\circ} 261$. 
Du fait de ces maladresses et de cette impréparation, l'essai de la suppression de cette dernière qui se présentait comme l'amorce d'une réorganisation administrative du royaume s'apparenta davantage, à une réaction de mauvaise humeur de la part de la monarchie, mécontente de ne pouvoir faire payer les officiers de finances et révéla, du fait de son échec final, une certaine impuissance de l'État à transformer profondément ses structures. Divers indices montrent pourtant que l'opération ne fut pas totalement sans lendemains. Malgré son recul, le roi continua en effet à exercer une surveillance étroite sur l'activité des gens des comptes. Au même moment où il nommait de nouveaux officiers à la Chambre, il envoya une nouvelle commission à Nantes pour obtenir un état au vrai de toutes les sommes dues par les receveurs de finances en Bretagne, et ce depuis la période ducale. Dans les lettres remises à son commissaire - il s'agissait encore du secrétaire du duc d'Anjou, Belvéder -, il ordonnait aux gens des comptes de se consacrer prioritairement à cette tâche et menaçait :

"Vous avons déffendu et déffendons de désemparer le service que vous nous devez en la Chambre... sur peine de privation de vos offices et privation de vos droitz et gaiges, saichant très bien qu'il seroit impossible en trois moys pouvoir mectre fin à tant d'arréraiges, lesquelz ne sont demeurez en arrière que par votre faulte et négligence, à quoy n'avons trouvé meilleur ni plus expédiant moyen que de vous astreindre à ce service jusques à ce que lesdites négligences soient réparées par la dilligence de ceux qui les ont commises $^{69}$."

Un an plus tard, en novembre 1569, de nouveaux commissaires intervenaient à la Chambre pour savoir de quelle autorité les gens des comptes accordaient des prorogations de feux de fouage en Bretagne et pour s'informer des tarifs qu'ils pratiquaient en la matière ${ }^{70}$, laissant deviner, là encore, que leurs pratiques restaient suspectes aux yeux des hommes au pouvoir ${ }^{71}$. Une réflexion continua par ailleurs à être menée sur l'organisation administrative de la province : l'idée de réunir parlement et Chambre des comptes à Rennes fut ainsi envisagée en 1572 afin de faciliter la collaboration des cours souveraines en matière de contrôle des finances ${ }^{72}$.

69. Idem, B $586 \mathrm{f}^{\circ}$ 67-68, mention du 10 juillet 1568 . Devant le refus de la Chambre de se soumettre immédiatement à ses ordres, Charles IX renvoya Belvéder à Nantes en novembre 1568 en donnant l'ordre au président François Le Bloy et au maître Jean Sorée de l'aider à remplir sa tâche. Là encore les gens des comptes firent traîner les choses en jouant sur des règles de procédure, montrant que pour eux l'heure de la soumission sans discuter à la volonté royale était passée, Arch. dép. de Loire-Atlantique, B 586 f $^{\circ} 79$.

70. Arch. dép. de Loire-Atlantique, B 587 f $^{\circ} 37$, mention du 10 novembre 1569. Les commissaires étaient René de Bourgneuf, président du parlement de Bretagne et François Gaudart, sieur de la Fontaine, maître des comptes à Paris.

71. La Chambre répondit au roi par une longue lettre de remontrance où elle fit un historique des circonstances qui lui avaient permis d'accorder les prorogations de feux, Arch. dép. de Loire-Atlantique, B $587 \mathrm{f}^{\circ} 66$.

72. Nicolas TRAVERS, Histoire civile, politique et religieuse de la ville et du comté de Nantes, Nantes, 1836-1841, t. 2, p. 438. Le projet de réunir parlement et Chambre des comptes à Rennes était encore évoqué dans les années 1580. 
L'opposition de la municipalité nantaise ne permit pas au projet d'aboutir mais ne mit pas fin aux projets de réforme puisque c'est au cours de cette même année 1572 que le roi décida le doublement du personnel de la Chambre et modifia le rythme de ses travaux en imposant le système des deux semestres. Moyen pour faire rentrer de nouveaux deniers dans les caisses royales, ce procédé permettait aussi d'espérer un fonctionnement plus efficace de l'institution et constituait en cela le terme de la période mouvementée qui avait débuté avec le passage du roi à Châteaubriant en octobre 1565.

L'étude de la crise de la Chambre des comptes au cours des années 1560 offre donc l'exemple d'un pouvoir tentant de réformer son mode de fonctionnement et de mieux contrôler son personnel. Les conditions du changement semblaient réunies du fait des dysfonctionnements institutionnels constatés, des insuffisances d'officiers trop éloignés de l'idéal du parfait magistrat, de la volonté de l'État enfin de se présenter en modèle et en arbitre en une période où la crise des consciences était particulièrement aiguë. La réforme envisagée connut bien un début d'application, ce qui empêche de la considérer comme un simple projet chimérique, mais elle n'aboutit pas. Poids de la conjoncture politique, insuffisante prise en compte des réalités locales, incapacité de l'État à se passer des agents incriminés expliquent l'échec final de Michel de L'Hospital. Celui-ci avait su dénoncer les maux mais se montra incapable de trouver les remèdes appropriés pour y mettre fin. Après sa mise à l'écart de la chancellerie, l'État n'abandonna pas toute idée de changement mais lia désormais celle-ci à une augmentation de ses effectifs, renonçant ainsi à l'idéal d'un corps de magistrats réduits et exemplaires. En ce sens, la crise qui marque les Chambres des comptes provinciales dans les années 1560 peut apparaître comme un tournant à partir duquel l'État cessa d'exercer un contrôle étroit sur ses agents, privilégia l'argent sur le talent et s'abandonna de plus en plus à la vénalité. Dans le cas breton, cela permit à la cour des comptes de s'inscrire définitivement dans le paysage institutionnel de la province et de survivre pendant plus de deux siècles puisqu'elle ne disparut définitivement qu'en 1791. 
Dominique LE PAGE

Annexe - Le personnel de la Chambre des comptes de Bretagne en 1564

\begin{tabular}{|c|c|c|c|c|}
\hline Fonctions & Officier & Date de réception & Date de sortie & Origine \\
\hline $\begin{array}{l}\text { Premier } \\
\text { président }\end{array}$ & Marc de Fortia & 5.5 .1551 & 9.5 .1573 & Touraine \\
\hline $\begin{array}{l}\text { Second } \\
\text { président }\end{array}$ & Claude Méaucé & 8.5 .1560 & 2.6 .1565 & Év. de Nantes \\
\hline Maître* & François Motay & 27.10 .1546 & av. le 23.3.1564 & Év. de Nantes \\
\hline Maître & Martin de Chaurais & 30.4 .1549 & 1565 & Blésois \\
\hline Maître & Pierre Gautier & 5.5 .1552 & 27.2 .1580 & Év. de Nantes \\
\hline Maître & René Bonnier & 19.4.1554 & Vers 1565 & Év. de Rennes \\
\hline Maître & Martin de Beaune & 21.5.1555 & 3.2 .1578 & Touraine \\
\hline Maître & Jean Sorée & 3.12 .1555 & 11.2 .1581 & Touraine \\
\hline Maître & Pierre de Francheville & 15.1 .1557 & 15.6 .1580 & Év. de Vannes \\
\hline Auditeur** & Pierre Riou & 20.11 .1540 & 1564 ou 1565 & Év. de Nantes \\
\hline Auditeur & Geoffroy de Callac & 17.7.1546 & 1564 ou 1565 & Év. de Vannes \\
\hline Auditeur & Yves de La Tullaye & 23.4.1555 & 13.01 .1571 & Év. de Nantes \\
\hline Auditeur & Tristan de St-Martin & 15.10 .1557 & 1564 ou 1565 & Év. de Nantes \\
\hline Auditeur & Golven de Kerchrist & 18.12 .1558 & 1566 & Év. de Léon \\
\hline Auditeur & Pierre Ménardeau & 7.4 .1559 & 22.4 .1572 & Év. de Nantes \\
\hline Auditeur & René Hachon & 15.6 .1559 & avril 1572 & Év. de Nantes \\
\hline Auditeur & Jean Guilloppe & 28.10 .1560 & 1601 & Év. de Nantes \\
\hline $\begin{array}{l}\text { Procureur } \\
\text { général }\end{array}$ & $\begin{array}{c}\text { Guillaume } \\
\text { de Francheville }\end{array}$ & 11.2 .1557 & 1575 & Év. de Vannes \\
\hline $\begin{array}{l}\text { Garde } \\
\text { des livres }\end{array}$ & Guillaume Meneust & 28.8 .1532 & 1570 & Év. de Nantes? \\
\hline Payeur & Jean Avril & 1548 & 1586 & Év. de Nantes \\
\hline
\end{tabular}

* Du fait du non-remplacement de Guillaume Morin décédé au début des années 1560, les maîtres ne sont plus que sept.

** Les auditeurs n'étaient plus que huit en raison de la mort de Julien Coué.

\section{RESUME}

Suite au tour de France effectué par Charles IX de 1564 à 1566, la monarchie tenta de supprimer les Chambres des comptes provinciales et de centraliser les moyens du contrôle financier à Paris. L'étude du cas breton montre que la réforme connut un début d'application, contrairement à ce que l'on a longtemps pensé, mais qu'elle échoua du fait d'une conjoncture politique défavorable et d'une insuffisante prise en compte des réalités locales.

\section{ABSTRACT}

In the wake of Charles IX's tour of France from 1564 to 1566, the monarchy attempted to suppress provincial Chambers of account and to centralize the means of financial control in Paris. The study of the Breton case shows that contrary to what was long thought, the reform was applied at the beginning but failed afterwards because the political situation was unfavourable and local realities were not enough taken into account. 